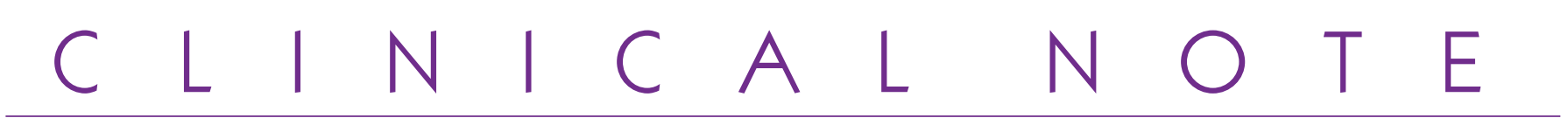

\title{
Sucking and swallowing
}

\author{
P. Fellus
}

Specialist in dentofacial orthopedics

Doctor of odontologic science

Consultant, Robert Debré University Hospital Center, Paris, France

\section{ABSTRACT}

The absence of a bilabial contact forbids any possibility of realizing a negative pressure inside the oral cavity. It implies that the patient cannot resort to the suction gulp when he swallows his saliva. The molar occlusion will allow the lingual dome to play its role of functional matrix and to establish a nasal breath. Froggy Mouth ${ }^{\circledR}$ is a small removable appliance particularly adapted to the young patients. It is carried 15 minutes a day in front of the television, without damaging the sleep quality.

It allows the stimulation of the neural circuits which manage the automatic movements and not the voluntary movements.

\section{KEYWORDS}

Sucking deglutition, swallowing deglutition, neural circuits of voluntary movements, neural circuits of automatic movements, Froggy Mouth

While, during gestation, the fetus was in an aquatic environment at constant temperature and with continuous nutrient input, delivery propels it into an aerial environment in which it must breathe and feed by itself. Fortunately, it has been training in utero since the $11^{\text {th }}$ week, swallowing amniotic fluid, to develop a functional unit enabling it, in the first minutes of life, to find the breast and meet its nutritional needs.

This swallowing program, of paleocortical origin, remains physiological as long as the mouth has no teeth ${ }^{2,3}$; the tongue fills the oral cavity completely, in contact laterally with the cheeks and anteriorly with the labial mucosa. The airtight joint required for suction is created by contracting the lips. Once the dental system, however, develops, alternating unilateral mastication begins slowly to mature in successive stages, from soft to compact foods ${ }^{1}$.

Persistent suction-gulp slows down these developments and labio-jugal muscle activity impairs physiological growth, causing variable 3-dimensional skeletal disorders. 


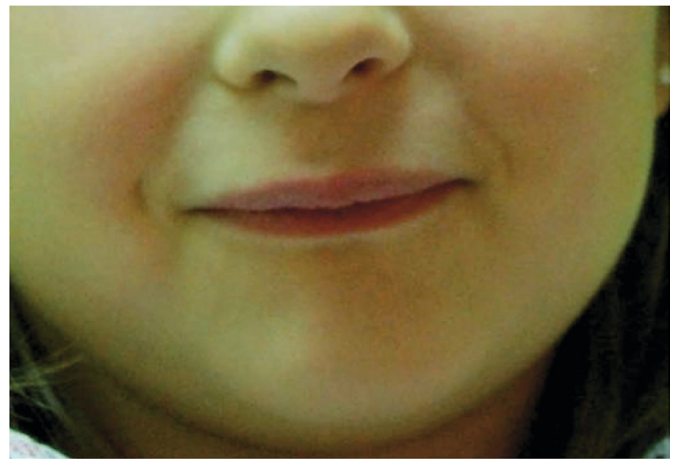

Figure 1

Lips contracted, and depressor labii inferioris and chin also: the suction-gulp program will be launched.

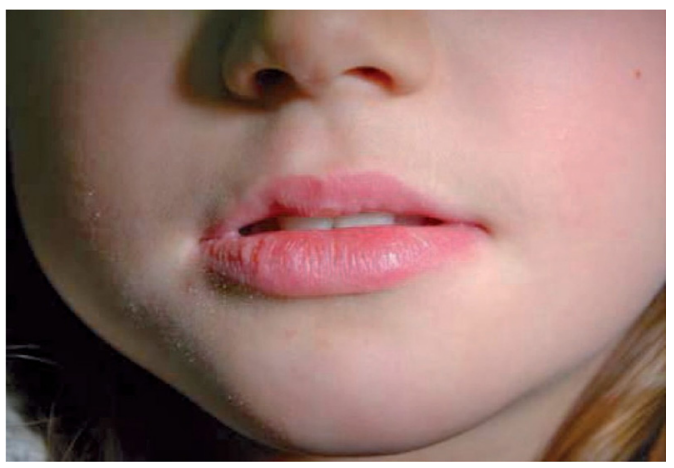

Figure 2

Lips apart; a tight lip joint is impossible and the new program will govern the swallowing sequence.

A new swallowing program is therefore required, and develops spontaneously in young children, $60 \%$ of whom discover a new way of swallowing by 4 years of age, and will be among those $50 \%$ of children who never require orthodontic treatment.

They spontaneously discover the need for a high posterior lingual dome position, achieved by tensing the styloglossus, a muscle which also narrows the tongue, bringing the apex of the tongue into a physiological area without sensory reference. There is thus posteriorization of the baseline motor configuration, from exofacial to endofacial.

If this does not happen naturally, then, like with a computer, a new program will have to be installed ${ }^{3}$. This is what the practitioner or speechtherapist does, in a series of codified exercises to program a new praxis $^{6}$ in the short-term memory circuits. This then has quickly to become longterm. The child at this point has two swallowing programs, as, just like in a computer, a program cannot actually be deleted from the hard drive or cortex. The old program just has not to be used: the child should stop clicking on that icon but rather on the new one. The old icon, is for "My lips are contracted" (Fig. 1), and the new one means "my lips are relaxed (Fig. 2) and my back-teeth are touching". If, when the child swallows saliva, the lips are contracted by facial nerve activity, the old program will automatically be switched on. If, on the other hand, the lips are relaxed and the teeth closed together under trigeminal control, the right program will be launched.

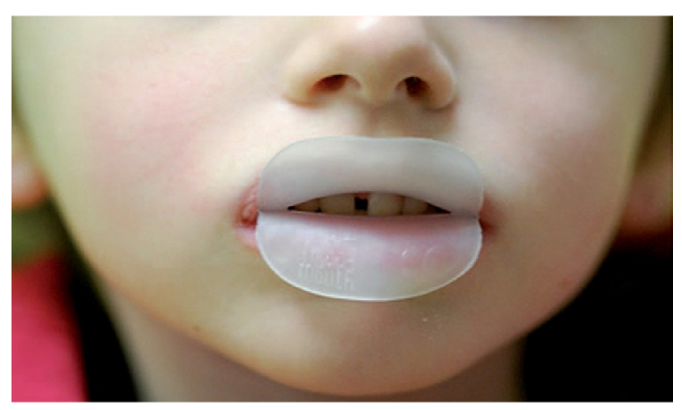

Figure 3

Froggy Mouth ${ }^{\circledR}$, worn for 15 minutes a day in front of the TV, facilitates automatic acquisition of the new swallowing program which is spontaneously established in $60 \%$ of children by the age of 4 years. 


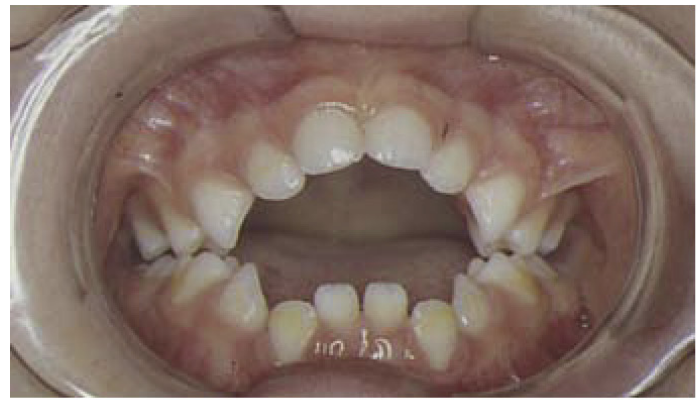

a

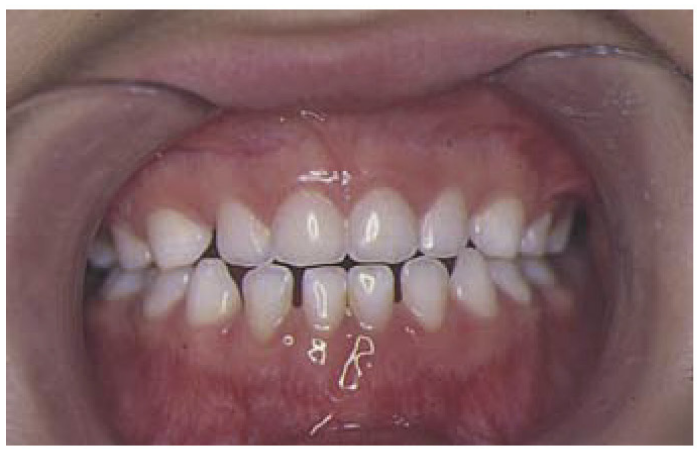

$C$

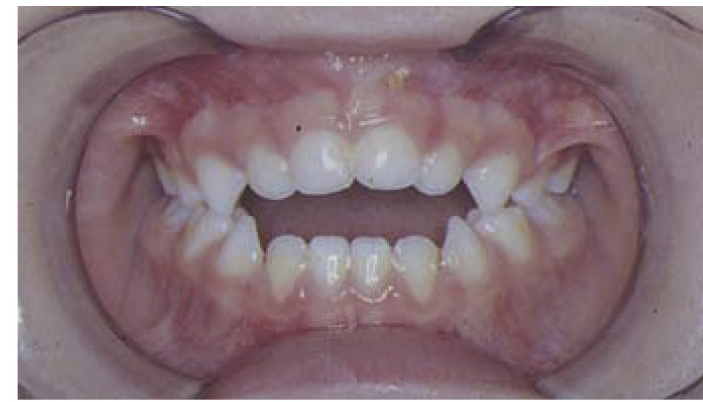

$\mathrm{b}$

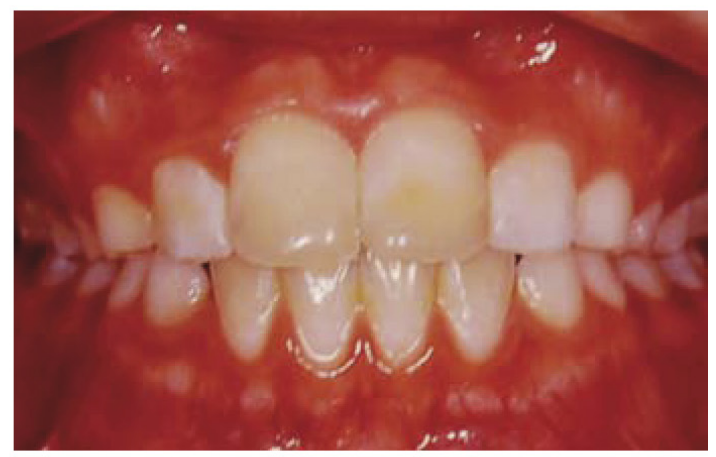

$\mathrm{d}$

Figure 4

a) Wide incisor gap caused by use of a dummy. b) Spontaneous improvement after withdrawal of dummy. c) Swallowing rehabilitation provides complete correction of dysmorphosis. d) Occlusion, with no further intervention, 3 years later.

Thus, swallowing rehabilitation needs to be based not on control of voluntary movements but on stimulating the subcortical connections that are functional in all children by 4 years of age and on activating the inhibition of the suctiongulp circuit5. This approach is founded on the work of Eric Kandel, 2000 winner of the Nobel Prize for medicine.

Froggy Mouth ${ }^{\circledR}$ is a device that helps choose the right icon (Fig. 3). It is placed sagittally between the lips and teeth, separating the upper from the lower lip. Absence of bilabial contact prevents any negative pressure within the oral cavity and thus any use of suction-gulp to swallow saliva.
Initially, it should bewornfor 15 minutes a day, in front of the TV or computer screen, so as to activate the neurologic circuits governing automatic movement under the control of the trigeminal nerve, which also controls the respiratory centers ${ }^{4}$, promoting recovery of nasal breathing. This very short daily practice does not interfere with the child's sleep, which is very important, and the playful activity that continues during the exercise acts as a reward from the point of view of the limbic system.

The approach is just as well suited for older children: acquisition may take longer after the physiological age. 


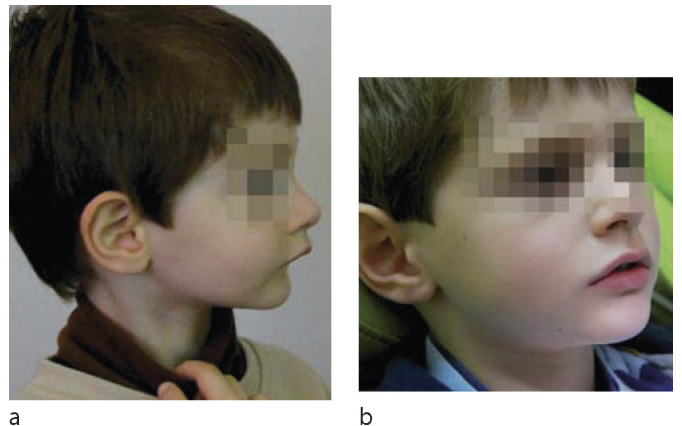

Figure 5

a) Retromandibulism in an inveterate thumb-sucker, aged 3; b) end of treatment.

But none of this can happen unless suction habits, which are physiological during the first months of life, have disappeared (Figs. 4, 5).

This is why it is important to be sure than baby-bottles and dummies have been withdrawn.

During the first year of life, a dummy can significantly reduce the risk of sudden infant death and facilitate sleeping, but should ideally be withdrawn by 1 year of age. A Swedish study published 30 years ago reported that use of dummies beyond 2 years of age impaired communication and language acquisition.

But we should perhaps go further and analyze the psychological impact of dummies. It is not the same for thumbsucking: many in utero ultrasound images show that digital sucking is discovered early on; during childhood, children manages this body part themselves, on the road to autonomy.

Dummies, in contrast, are foreign bodies introduced by parents or care-givers, and are integrated psychologically as part of the child's outside world.

Worn-out dummy, dummy lost: the child, to get satisfaction, is dependent on a third party (here, a parent).

Dummies should have disappeared by 2 years of age at the latest, as should feeding bottles. Withdrawal from thumb-sucking, on the other hand, can await 4 years.

Beyond this age, withdrawal can be helped by wearing a very simple intraoral device which, by altering the afferent message reaching the brain, enables a new efferent message to be formed. In 95\% of cases, thumbsucking is a question of habit, and the present protocol enables spontaneous cessation without frustration, and is thus highly preferable to behavioral protocols requiring grids to be filled out with alternating suns and clouds - with feelings of guilt liable to arise in case of failure. For the $5 \%$ of children who go on sucking their thumbs despite wearing the device, sucking is not a "habit" but a "need"; this symptom may not always be serious, but it is always significant. A psychologist or child-psychiatrist should be systematically consulted in these rare cases, to have a differential diagnosis between delayed affective development and a more severe issue of psychological origin.

Conflicts of interest: The author is President of Orthopolis SAS, which holds the patent for Froggy Mouth. 


\section{REFERENCES}

1. Couly G. Les oralités humaines. Doin, 2010.

2. Deffez JP, Girard C, Fellus P. Rééducation de la déglutition salivaire. Éditions CdP, 1995.

3. Fellus P. Orthodontie précoce en denture temporaire. Éditions CdP, 2003.

4. Guyton A. Basic neurosciences: anatomy and physiology. Philadelphia: Saunders Company, 1991.

5. Kandel E. À la recherche de la mémoire. Une nouvelle théorie de l'esprit. Odile Jacob Sciences, 2007.

6. Piaget J. Les praxies chez l'enfant. Revue de neurologie 1960;102(6):551-566. 